\title{
Dissipative Solitary Waves in Granular Crystals
}

\author{
R. Carretero-González, ${ }^{1}$ D. Khatri, ${ }^{2}$ Mason A. Porter, ${ }^{3}$ P. G. Kevrekidis, ${ }^{4}$ and C. Daraio ${ }^{2, *}$ \\ ${ }^{1}$ Department of Mathematics and Statistics, San Diego State University, San Diego, California 92182-7720, USA \\ ${ }^{2}$ Graduate Aeronautical Laboratories (GALCIT) and Department of Applied Physics, California Institute of Technology, \\ Pasadena, California 91125, USA \\ ${ }^{3}$ Oxford Center for Industrial and Applied Mathematics, Mathematical Institute, University of Oxford, OXI 3LB, United Kingdom \\ ${ }^{4}$ Department of Mathematics and Statistics, University of Massachusetts, Amherst, Massachusetts 01003-4515, USA
}

(Received 12 August 2008; published 16 January 2009)

\begin{abstract}
We provide a quantitative characterization of dissipative effects in one-dimensional granular crystals. We use the propagation of highly nonlinear solitary waves as a diagnostic tool and develop optimization schemes that allow one to compute the relevant exponents and prefactors of the dissipative terms in the equations of motion. We thereby propose a quantitatively accurate extension of the Hertzian model that encompasses dissipative effects via a discrete Laplacian of the velocities. Experiments and computations with steel, brass, and polytetrafluoroethylene reveal a common dissipation exponent with a materialdependent prefactor.
\end{abstract}

DOI: 10.1103/PhysRevLett.102.024102

PACS numbers: 45.70.-n, 05.45.Yv, 43.25.+y, 46.40.Cd

Introduction.-Since the advent of the famous FermiPasta-Ulam model over 50 years ago, nonlinear oscillator chains have received a remarkable amount of attention in a wide range of physical settings [1]. Areas of intense theoretical and experimental interest over the past decade include (but are not limited to) DNA double-strand dynamics in biophysics [2], coupled waveguide arrays in nonlinear optics [3], breathing oscillations in micromechanical cantilever arrays [4], and Bose-Einstein condensation in optical lattices in atomic physics [5].

Within this general theme of interplay between nonlinearity and discreteness, one of the key subjects has been the study of 1D granular materials, which consist of chains of interacting particles that start from point contact with each other and deform elastically when compressed. In contrast to classically studied disordered granular media, highly packed granular lattices have negligible frictional and rotational dynamics, in favor of axial stress propagation [6,7]. The highly nonlinear dynamic response of such "crystals" has been the subject of considerable attention [6,8-23]. Additionally, granular crystals can be created from numerous material types and sizes, which makes their properties extremely tunable [8-10]. This flexibility is valuable not only for basic studies of the underlying physics but also in potential applications such as shock [24] and energy absorbing layers [13,18,20,21], sound focusing devices (tunable acoustic lenses and delay lines), actuators [25,26], sound absorption layers, and sound scramblers $[11,12,23]$.

While the standard Hertzian force model has been used extensively in most dynamical investigations and is now textbook material $[8,27]$, recent experimentally motivated investigations have illustrated the challenging need to include dissipation effects [24,28-30]. Dissipative terms related to friction [31], plasticity [32], viscoelasticity [33], and viscous drag $[23,30]$ have been proposed to model particle collisions $[6,7,27]$. However, none of these models captures both qualitatively and quantitatively the decay and wave shape of the highly nonlinear solitary waves observed experimentally. It is this important experimental and theoretical aspect of packed granular lattices that we aim to tackle in this Letter through the combination of modeling, numerical and physical experiments, and a detailed comparison thereof. Based on the earlier propositions of Refs. [6,7,24,30], we illustrate the prevalent nature of dissipation in the form of a discrete Laplacian in the velocities with uniform exponent and a material-dependent prefactor. The broad interest of our findings stems not only from their general nature for granular crystals of different materials but also from the significance of similar models in other fields, such as 1D lattice turbulence [34].

Experimental setup.-We assembled a monodisperse chain of $N$ beads (here we report results for $N=70$ but we performed experiments for up to $N=188$ with similar results) of different materials (see Table I) with radius $R=$ $2.38 \mathrm{~mm}$ in a horizontal setup [see Fig. 1(a)] arranged in a four-garolite rod stand. (To ensure contact between the particles, the guide was tilted at $4^{\circ}$.) To directly visualize the waves, we embedded calibrated piezosensors $(R C \sim$ $10^{3} \mu$ s, Piezo Systems, Inc; see Fig. 1(b) of Ref. [11])

TABLE I. Material properties (mass $m$, elastic modulus $E$, and Poisson ratio $\nu$ ) for stainless steel [35,36], PTFE [11,37,38], and brass [39]. The last two columns present our best estimates, together with their standard deviation, of the dissipation coefficients $(\alpha, \gamma)$.

\begin{tabular}{llcccc}
\hline \hline Material & $m(\mathrm{~g})$ & $E(\mathrm{GPa})$ & $\nu$ & $\alpha$ & $\gamma$ \\
\hline Steel & 0.45 & 193 & 0.30 & $1.81 \pm 0.25$ & $-5.58 \pm 1.30$ \\
PTFE & 0.123 & 1.46 & 0.46 & $1.68 \pm 0.16$ & $-1.56 \pm 0.19$ \\
Brass & 0.48 & 103 & 0.34 & $1.85 \pm 0.13$ & $-6.84 \pm 0.66$ \\
\hline \hline
\end{tabular}



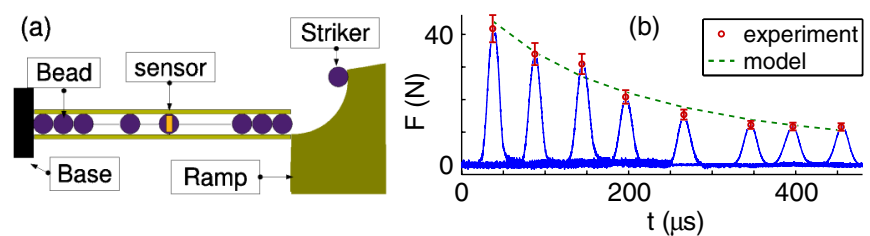

FIG. 1 (color online). (a) Schematic diagram of the experimental setup. (b) Solitary wave decay in a chain composed of 70 steel particles impacted by a steel bead with $v_{\text {imp }}=v_{1}$. The (blue) solid curves correspond to the recordings for sensors placed in particles 9, 16, 24, 31, 40, 50, 56, and 63 .

inside selected particles, as described in Refs. [10-13]. We generated solitary waves by impacting the chain with a striker (identical to the particles in the chain) launched along a ramp. We calculated the impact velocities $v_{\text {imp }}$ (in $\mathrm{m} / \mathrm{s}$ ) using a high-speed camera at the end of the ramp: $v_{1,2}=1.77, v_{3,4}=1.55, v_{5,6}=1.40, v_{7,8}=1.04$, and $v_{9,10}=0.79$.

Model.-We model a dissipative chain of $N$ spherical beads as a 1D lattice with Hertzian interactions [8]:

$$
\ddot{y}_{n}=A\left(\delta_{n}^{3 / 2}-\delta_{n+1}^{3 / 2}\right)+\gamma_{S}\left|\dot{\delta}_{n}-\dot{\delta}_{n+1}\right|^{\alpha},
$$

where $s \equiv \operatorname{sgn}\left(\dot{\delta}_{n}-\dot{\delta}_{n+1}\right), \quad A \equiv E \sqrt{2 R} /\left[3 m\left(1-\nu^{2}\right)\right]$, $n \in\{1, \ldots, N\}, y_{n}$ is the deviation of the $n$th bead from its equilibrium, $\delta_{n} \equiv \max \left\{y_{n-1}-y_{n}, 0\right\} \quad$ for $n \in$ $\{2, \ldots, N\}, \delta_{1} \equiv 0, \delta_{N+1} \equiv \max \left\{y_{N}, 0\right\}, E$ is the Young's (elastic) modulus of the beads, $\nu$ is their Poisson ratio, $m$ is their mass, and $R$ is their radius. The particle $n=0$ represents the striker. Dissipation is incorporated by using a phenomenological force with prefactor $\gamma<0$, between adjacent beads that depends on their relative velocities (in particular, on the "discrete Laplacian" in the velocities), generalizing earlier models (with dissipation exponent $\alpha=1$ specifically) for dry granular matter [30].

In contrast to previous works that $a$ priori assume that $\alpha=1$ (i.e., that model dissipation using a linear dashpot) [30], we determine both $\alpha$ and $\gamma$ by directly comparing experimental and numerical results. The general coefficient $\alpha$ is thus a phenomenological parameter derived from the best fitting. We introduce the absolute value and the sign parameter $s$ into (1) to ensure that genuine dissipation is guaranteed irrespective of the sign of the relative velocities between consecutive beads. The units of $\gamma$ would depend on the value of $\alpha$ and, accordingly, are more properly investigated in dynamic models that incorporate dissipation based on detailed measurements of restitutive losses that cannot currently be achieved experimentally [6]. Importantly, the value we obtain for $\alpha$ differs decidedly from the coefficients used in previous modeling attempts [30] (see the discussion below).

Determining the dissipation coefficients.-We now determine the "optimal" dissipation coefficients $(\alpha, \gamma)$ from the experimental data for different materials and different configurations. The experimental data consist of the time series of the force through each sensor. We optimize the pair $(\alpha, \gamma)$ by minimizing the following two differences between numerics and each particular experiment:

$$
\begin{gathered}
D(\alpha, \gamma)=\frac{1}{N} \sum_{n=1}^{N} \frac{\left|F_{m}^{\operatorname{expt}}(n)-F_{m}^{\mathrm{num}}(n)\right|}{\bar{F}_{m}^{\operatorname{expt}}}, \\
\Delta_{n}(\alpha, \gamma)=\frac{1}{T} \int_{t_{i}}^{t_{f}} \frac{\left|F^{\operatorname{expt}}(t ; n)-F^{\mathrm{num}}(t ; n)\right|}{\bar{F}^{\operatorname{expt}}(n)} d t,
\end{gathered}
$$

where $\quad \bar{F}_{m}^{\text {expt }} \equiv(1 / N) \sum_{n=1}^{N} F_{m}^{\text {expt }}(n), \quad \bar{F}^{\operatorname{expt}}(n)=$ $\int_{t_{i}}^{t_{f}} F^{\operatorname{expt}}(t ; n) d t, F(t ; n)$ is the time series data of the force through the $n$th sensor [see Fig. 1(b)], and $F_{m}(n)=$ $\max _{t}\{F(t ; n)\}$ is the maximum force recorded by the $n$th sensor over the recording time $\operatorname{span}\left[t_{i}, t_{f}=t_{i}+T\right]$, where $T$ is typically about $100 \mu \mathrm{s}$. The superscripts "expt" and "num" denote, respectively, the experimental and numerical data. The function $D(\alpha, \gamma)$ measures the "distance" between the numerics and the experiment using the maxima of the forces through all sensors of the experiment. The function $\Delta_{n}(\alpha, \gamma)$ measures the difference between experimental and numerical pulse shapes that go through the $n$th sensor. In order to avoid biasing $\Delta_{n}(\alpha, \gamma)$ with the difference in force magnitude [which is already taken into account when optimizing $D(\alpha, \gamma)$ ], we rescale the experimental data so that the numerical and experimental maxima match before we compare wave forms. That is, $F^{\operatorname{expt}}(t ; n) \rightarrow F^{\operatorname{expt}}(t ; n) F_{m}^{\text {num }}(n) / F_{m}^{\text {expt }}(n)$. Figures $2($ a) and 2(b) depict, respectively, the differences $D(\alpha, \gamma)$ and $\Delta_{n}(\alpha, \gamma)$ in a particular $(\alpha, \gamma)$ range for a steel chain using a sensor placed towards the end of the chain. As can be observed from these panels, the optimization of the force maxima $F_{m}^{\text {num }}$ [Fig. 2(a)] and the force pulse shape [Fig. 2(b)] are not sufficient on their own to determine the dissipation parameters. However, it is meaningful (and well defined) to optimize force maxima and pulse shape together by taking the intersection between the minima of each case (see the point at the intersection of the solid curves and dashed curves). For experiment $j$ (with impact velocity $\left.v_{j}\right)$, we average the parameter pair $\left(\alpha_{j}, \gamma_{j}\right)$ over four sensors located throughout the bead chain. Finally, we average $(\alpha, \gamma)=\frac{1}{N_{e}} \sum_{j=1}^{N_{e}}\left(\alpha_{j}, \gamma_{j}\right)$ over the $N_{e}=10$ different experiments to obtain the optimal dissipation parameters $(\alpha, \gamma)$ and compute the standard deviation for the $N_{e}$ experiments.

We summarize our results, for three different set of experiments-using steel, Teflon [polytetrafluoroethylene (PTFE)], and brass beads-in the last two columns of Table I. In order to validate the results of the above optimization procedure a posteriori, we take the optimal dissipation parameters for the steel bead chain and compare the maximal forces obtained numerically with the experiments in Fig. 2(c). In Fig. 2(c), we show two typical examples (for impact velocities $v_{3}$ and $v_{8}$ ) and also plot the curves incorporating the standard deviation measured in our analysis. As can be clearly observed, all experimental data points fall well within the predicted region. To further 

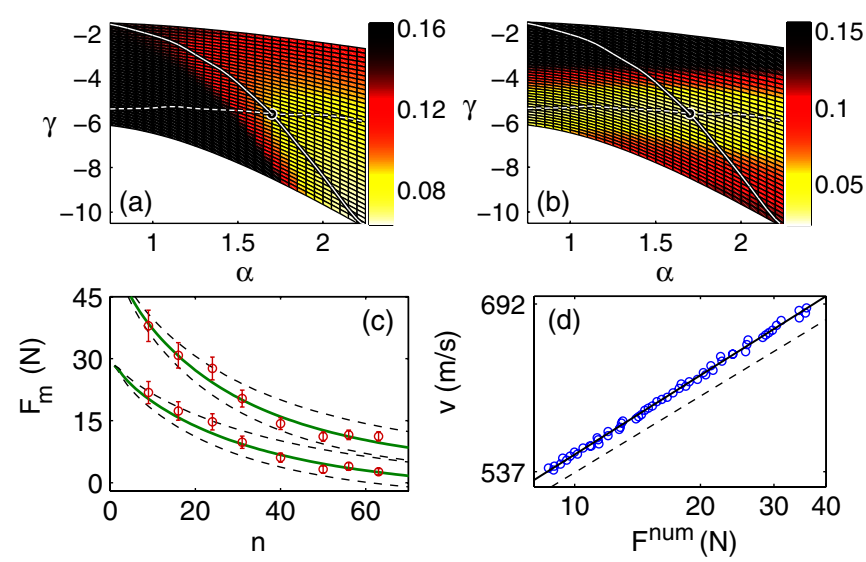

FIG. 2 (color online). Optimization of the dissipation coefficients $(\alpha, \gamma)$ for a steel chain. (a) Difference $D(\alpha, \gamma)$, as defined in Eq. (2), between the force maxima recorded in the experiment and our model. (b) Difference $\Delta_{n}(\alpha, \gamma)$, as defined in Eq. (3), in wave forms between the experiment and our model for sensor $n=56$. The solid curves and dashed curves correspond to the minima obtained from (a) and (b), respectively. (c) Maximum force $F_{m}^{\text {num }}(n)$ for experiments with $v_{\text {imp }}=v_{3}$ (top curves) and $v_{\text {imp }}=v_{8}$ (bottom curves, displaced by 5 units for clarity). The (red) circles correspond to the experiment, and the (green) thick curves give the numerical best fit with $(\alpha, \gamma)=(1.81 \pm$ $0.25,-5.58 \pm 1.30)$. The dashed curves correspond to the extreme cases using the standard deviation found in the optimal parameters. (d) Velocity of traveling front versus the maximum force (in a log-log plot). The solid curve represents the best linear fit, which gives $v \propto F_{m}^{0.17}$; we also show a dashed line with slope $1 / 6$.

validate our results, we compared the dependence of the pulse velocity $v$ against the maximal force $F_{m}^{\text {num }}$ in Fig. 2(d). This panel shows a typical example; we obtained similar results (not shown here) for the other configurations. The obtained exponent is extremely close to the theoretical value of $1 / 6$ (shown by the dashed line) [8].

In order to gain a deeper understanding of the role of the dissipation exponent $\alpha$, we depict in Fig. 3 the pulse shape for two sensors in the steel chain (one near the beginning of
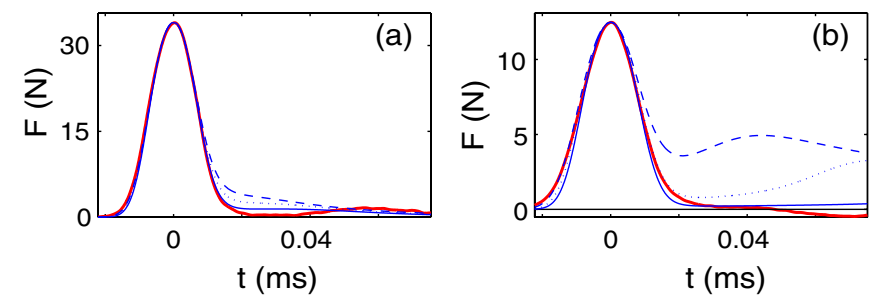

FIG. 3 (color online). Force versus time for the steel chain with $v_{\text {imp }}=v_{2}$ through sensors at positions (a) $n=16$ and (b) $n=56$. The (red) thick solid curve depicts the (smoothed; see text) experimental series, and the thin (blue) dashed curves, dotted curves, and solid curves, respectively, show the numerics with $(\alpha, \gamma)=(1,-5.5),(1.4,-6)$, and $(1.81,-5.58)$. The last case corresponds to the best fit (see text) for the dissipation parameters for the chains of steel beads. the chain and the other one near the end). We depict the experimental pulse (smoothed by nearest-neighbor averaging) with the (red) solid curve. The thin (blue) curves, depict three numerical runs using three different pairs $(\alpha, \gamma)$ along the minimum curve [shown by a solid curve in Fig. 2(a)].

It is interesting to note that for all materials tested, higher impact velocities correspond to a faster initial decay as compared to the latter part of the chain (probably related to the initiation of plasticity at the contact). Also, by comparing the wave decay in chains composed of steel and Teflon (or brass) beads, a faster and more pronounced energy loss is evident for the softer beads. To understand this dissipation physically, one should explore a more detailed analysis of the contact plasticity, inelastic restitution, and hydrodynamic drag.

Note that the optimal dissipation exponent $\alpha$ for the three material types considered is consonant with a value close to $\alpha=1.75$. This indicates the prevalence of the phenomenological damping introduced in Eq. (1), which is one of the principal findings of this Letter. It is important to point out the disparity of this optimal exponent from earlier investigations, which focused on the (linear dashpot) case of $\alpha=1[24,30,34]$. On the other hand, the dissipation prefactor $\gamma$ naturally does depend on the material. For steel and brass, which have similar material properties, $\gamma$ is also similar (steel has $\gamma=-5.58$ and brass has $\gamma=-6.84$ ). However, for Teflon, as can be anticipated from the much lower elastic modulus $E$, the prefactor $\gamma$ is significantly smaller $(\gamma=-1.56)$.

We show typical examples of the results for Teflon (left column) and brass (right column) in Fig. 4. The top panels depict the maximal force through the chain using the optimal dissipation parameters. Note in the pulse shape results (bottom panels) for Teflon and brass that low dissipation exponents $\alpha$ tend to overestimate the size of the secondary pulse hump. Another relevant observation, in connection with its much smaller dissipation prefactor $\gamma$, is that chains of Teflon beads may offer the first unambiguous observation of the secondary pulses [see Fig. 4(c)] argued in Ref. [30] to arise for weaker dissipation.

Conclusions. - In this Letter, we have offered for the first time a quantitative and systematic modeling attempt at the role of dissipation in granular crystals. Through detailed comparison of numerical simulations and experiments in a variety of materials (steel, Teflon, and brass), we have demonstrated a generic functional form of the dissipation, modeled by a phenomenological term based on the second difference of the velocities between adjacent beads (i.e., a discrete Laplacian) that is raised to a common exponent. This allowed us to augment the standard dynamical model based on Hertzian forces to encompass this dissipation effect in (optimal) quantitative agreement with our experiments. We found that the dissipation prefactor is material dependent and that the considerably weaker prefactor of Teflon (in comparison to brass and steel) allows one to observe unambiguously (and for the first time) secondary 

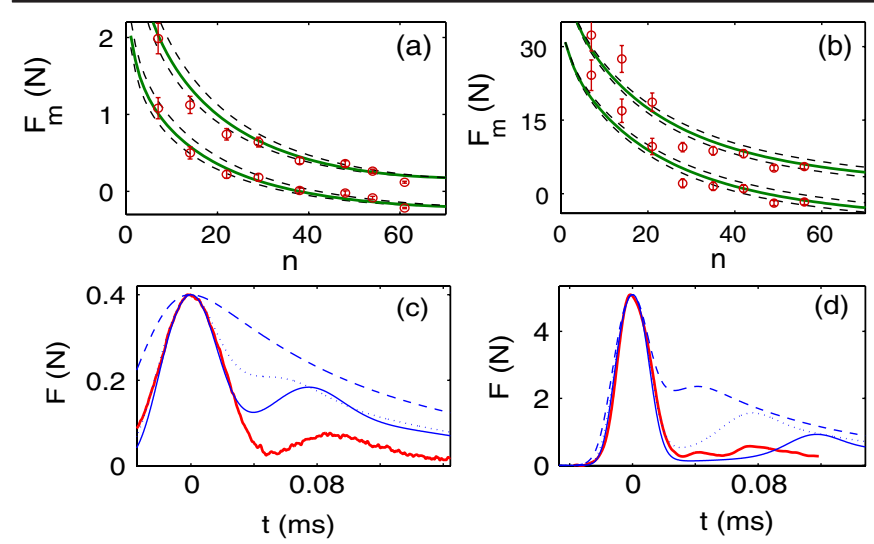

FIG. 4 (color online). Results for the Teflon (left column) and brass (right column) experiments. The top panels depict the same information as Fig. 2(c) for experiments with impact velocities (a) $v_{3}$ (top curves) and $v_{8}$ (bottom curves, displaced by 0.3 units for clarity) and (b) velocities $v_{3}$ (top curves) and $v_{6}$ (bottom curves, displaced by 7 units for clarity). The best fit for the dissipation parameters for Teflon and brass are, respectively, $\quad(\alpha, \gamma)=(1.68 \pm 0.16,-1.56 \pm 0.19)$ and $(\alpha, \gamma)=$ $(1.85 \pm 0.13,-6.84 \pm 0.66)$. The bottom panels show the same information as in Fig. 3. In (c), we depict the force versus time through the sensor at $n=38$ with $(\alpha, \gamma)=(1,-1.56)$, $(1.4,-1.56)$, and $(1.68,-1.56)$. In $(d)$, we show the same information for the sensor at $n=14$ with $(\alpha, \gamma)=(1,-5.5)$, $(1.4,-6)$, and $(1.85,-6.84)$.

pulses such as the ones proposed in Ref. [30]. Our study also provides a starting point for future quantitative investigations of this newly proposed model. For example, it would be worth examining the critical prefactor below which a secondary wave should be expected to emerge, the interplay of the role of dissipation and plasticity (and a quantitative incorporation of the latter) in the dynamics, and extensions of the present considerations to higherdimensional settings.

C. D. acknowledges support from NSF-CMMI 0825345, and P. G. K. acknowledges support from NSF-DMS, NSFCAREER, and the AvH Foundation. We thank Charles Campbell for useful discussions.

*Corresponding author.

daraio@caltech.edu

[1] E. Fermi et al., Los Alamos National Laboratory Technical Report No. LA-1940, 1955; D. K. Campbell et al., Chaos 15, 015101 (2005).

[2] M. Peyrard, Nonlinearity 17, R1 (2004).

[3] Y.S. Kivshar and G. P. Agrawal, Optical Solitons: From Fibers to Photonic Crystals (Academic, San Diego, 2003).

[4] M. Sato, B. E. Hubbard, and A. J. Sievers, Rev. Mod. Phys. 78, 137 (2006).
[5] O. Morsch and M. Oberthaler, Rev. Mod. Phys. 78, 179 (2006); V. A. Brazhnyi and V. V. Konotop, Mod. Phys. Lett. B 18, 627 (2004).

[6] S. Sen, J. Hong, J. Bang, E. Avalos, and R. Doney, Phys. Rep. 462, 21 (2008).

[7] H. M. Jaeger et al., Rev. Mod. Phys. 68, 1259 (1996).

[8] V.F. Nesterenko, Dynamics of Heterogeneous Materials (Springer-Verlag, New York, 2001).

[9] C. Coste, E. Falcon, and S. Fauve, Phys. Rev. E56, 6104 (1997).

[10] C. Daraio, V. F. Nesterenko, E. B. Herbold, and S. Jin, Phys. Rev. E 73, 026610 (2006).

[11] C. Daraio, V.F. Nesterenko, E. B. Herbold, and S. Jin, Phys. Rev. E 72, 016603 (2005).

[12] V.F. Nesterenko, C. Daraio, E. B. Herbold, and S. Jin, Phys. Rev. Lett. 95, 158702 (2005).

[13] C. Daraio et al., Phys. Rev. Lett. 96, 058002 (2006).

[14] E. Hascoët and H. J. Herrmann, Eur. Phys. J. B 14, 183 (2000).

[15] E. J. Hinch and S. Saint-Jean, Proc. R. Soc. A 455, 3201 (1999).

[16] M. Manciu et al., Physica (Amsterdam) 157D, 226 (2001).

[17] J. Hong and A. Xu, Appl. Phys. Lett. 81, 4868 (2002).

[18] J. Hong, Phys. Rev. Lett. 94, 108001 (2005).

[19] S. Job and F. Melo, Phys. Rev. Lett. 94, 178002 (2005).

[20] R. Doney and S. Sen, Phys. Rev. Lett. 97, 155502 (2006).

[21] L. Vergara, Phys. Rev. E 73, 066623 (2006).

[22] A. Sokolow et al., Europhys. Lett. 77, 24002 (2007).

[23] E. B. Herbold, V. F. Nesterenko, and C. Daraio, in Shock Compression of Condensed Matter, AIP Conf. Proc. No. 845 (AIP, New York, 2006), pp. 1523-1526.

[24] E. B. Herbold and V.F. Nesterenko, Phys. Rev. E 75, 021304 (2007).

[25] D. Khatri, C. Daraio, and P. Rizzo, Proc. SPIE Int. Soc. Opt. Eng. 6934, 69340U (2008).

[26] C. Daraio and P. Rizzo, U.S. provisional patent application filed 2008.

[27] K. L. Johnson, Contact Mechanics (Cambridge University Press, Cambridge, England, 1985).

[28] N. V. Brilliantov, F. Spahn, J. M. Hertzsch, and T. Pöschel, Phys. Rev. E 53, 5382 (1996); S. McNamara and E. Falcon, Phys. Rev. E 71, 031302 (2005).

[29] C. Brunhuber, F. G. Mertens, and Y. Gaididei, Phys. Rev. E 73, 016614 (2006).

[30] A. Rosas, A.H. Romero, V.F. Nesterenko, and K. Lindenberg, Phys. Rev. Lett. 98, 164301 (2007).

[31] W. J. Wang and Z. G. Zhu, Europhys. Lett. 82, 24004 (2008).

[32] I. Pane and E. Blank, Int. J. Solids Struct. 43, 2014 (2006).

[33] W. A. M. Morgado and I. Oppenheim, Phys. Rev. E 55, 1940 (1997).

[34] M. Peyrard and I. Daumont, Europhys. Lett. 59, 834 (2002).

[35] ASM Metals Reference Book (American Society for Metals, Metals Park, OH, 1983), 2nd ed.

[36] http://www.efunda.com.

[37] www.dupont.com/teflon/chemical/.

[38] W. J. Carter and S.P. Marsh, Los Alamos National Laboratory Technical Report No. LA-13006-MS, 1995.

[39] http://www.matweb.com. 